\title{
New Electronics for the Surface Detectors of the Pierre Auger Observatory
}

\author{
D. Nitz ${ }^{* a, b}$ for the Pierre Auger Collaboration ${ }^{{ }^{\dagger}}$ \\ ${ }^{a}$ Michigan Technological University, Houghton, Michigan, USA \\ ${ }^{b}$ Karlsruhe Institute of Technology, Karlsruhe, Germany \\ ${ }^{c}$ Observatorio Pierre Auger, Av. San Martín Norte 304, 5613 Malargüe, Argentina \\ E-mail: auger_spokespersons@fnal.gov \\ Full author list: http://www.auger.org/archive/authors_icrc_2019.html
}

\begin{abstract}
The surface detector array of the Pierre Auger Observatory consists of 1660 water-Cherenkov detector stations (WCDs) that sample the charged particles and photons of air showers initiated by energetic cosmic rays. Each station continuously samples signals from photomultiplier tubes (PMTs) viewing the water volume, and records $\mathrm{a} \approx 20 \mu$ s trace whenever a local trigger condition is satisfied. Absolute timing is provided by a GPS receiver on each station, and power is provided by a solar power system. The Observatory is currently implementing an upgrade, "AugerPrime". AugerPrime includes the addition of a small PMT to increase dynamic range, a plastic scintillator above each WCD to enhance the separation of electromagnetic and muon shower components, a radio detector to measure the radio emission of inclined air showers, and an infill of buried muon counters to provide additional cross checks. Consequently, new electronics to support these additional detectors is also being implemented. In addition to more measurement channels, the new electronics includes improved GPS receivers, higher sampling frequency, increased dynamic range, increased processing capability, and improved calibration and monitoring systems. This paper presents the design of the new electronics and discusses performance characteristics observed in laboratory measurements and engineering array data.
\end{abstract}

36th International Cosmic Ray Conference - ICRC2019

24 July - 1 August, 2019

Madison, Wisconsin, USA

\footnotetext{
*Speaker.

${ }^{\dagger}$ for collaboration list see PoS(ICRC2019)1177
} 


\section{Introduction}

The Pierre Auger Observatory [1] consists of 1600 water Cherenkov detectors (WCDs) on a $1500 \mathrm{~m}$ triangular grid covering $3000 \mathrm{~km}^{2}$ near Malargüe, Argentina. Another $60 \mathrm{WCDs}$ form an infill region. The array is overlooked by four fluorescence detector sites that each contain 6 telescopes viewing a $180^{\circ}$ azimuth by $30^{\circ}$ elevation field of view. Three additional telescopes at one of the sites can be tilted $30^{\circ}$ higher to view lower energy showers at their maximum and overlook a $30 \mathrm{~km}^{2}$ infilled surface array with a $750 \mathrm{~m}$ spacing. Extensive air showers (EAS) induced by ultra-high energy cosmic rays (UHECRs) are sampled at ground level by the Surface Detector (SD). The Fluorescence Detector (FD) measures the EAS development by detecting the nitrogen UV light produced by the shower's particles along their passage in the atmosphere. Additional instrumentation for R\&D on muon and radio-based detection is also located on the site.

In its first two decades, the Pierre Auger Observatory has made significant contributions to the study of UHECRs. In order to make further progress and more fully harness the power of statistics, the Auger Collaboration decided to to improve the sensitivity to composition of the SD. The Pierre Auger Observatory is therefore undergoing a significant upgrade of its experimental capabilities called AugerPrime. The AugerPrime upgrade will allow us to disentangle the muonic and electromagnetic components of extensive air showers [2,3,4], thereby enhancing the ability of the Observatory to study UHECR composition, composition-assisted anisotropies and hadronic interaction effects at the highest energies.

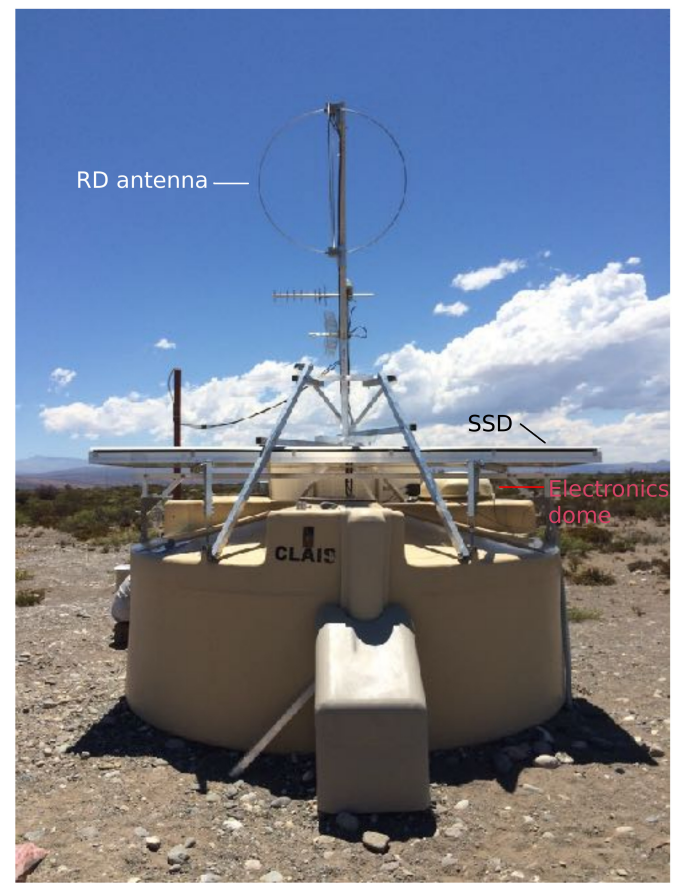

Figure 1: Photo of a prototype AugerPrime detector. Shown are the SSD (black annotation) atop the WCD along with a radio detector antenna (large circular antenna at highest point with white annotation). The UUB is hidden underneath the dome (red annotation) visible between the top of the WCD and bottom of the SSD on the right side of the top of the tank.

AugerPrime consists of a $3.8 \mathrm{~m} \times 1.3 \mathrm{~m}$ scintillator-based surface detector (SSD) and a radio detector (RD) [5] added atop each water Cherenkov detector. A full prototype station is shown in Fig. 1. This allows separation of the muonic and electromagnetic (EM) parts of the shower by exploiting the different responses of the various detector types to each component and therefore enhancing the composition sensitivity. The WCD has good sensitivity to both muonic and EM 
components while the SSD and RD are predominantly sensitive to the EM component. Shower universality and matrix methods are used to correct for differences in the response for each detector type. The SSD and RD are complementary in their solid angle coverage, with the SSD being optimized for more vertical showers, and the RD optimized for inclined showers with higher zenith angles [6]. A small PMT is added to the WCD to increase the dynamic range [7]. Improved support for the buried muon counters (AMIGA) [8] is also incorporated.

The surface detector electronics is being upgraded to support the detector enhancement. This involves replacing the main electronics board, the so-called Unified Board (UB) with an Upgraded Unified Board (UUB) [9]. In addition to providing support for the SSD, the RD, AMIGA, and the small PMT, the UUB includes faster and more precise ADCs and a faster central processor.

Backwards-compatibility with the current dataset is maintained by keeping the time-span of the PMT traces similar to that in the current electronics and by providing $20 \mathrm{MHz}$ cutoff digital filtering of the recorded traces in order to emulate the current triggers. Additional full bandwidth triggers are also implemented to enhance the triggering.

An AugerPrime engineering array (EA) of 12 stations has been operating at the Auger Observatory site since October, 2016 [10]. The EA is integrated in the standard Observatory's operation, with signal traces recorded by the SSD detectors and read out through the Auger communications network [11], packaged into events by the Central Data Acquisition system and sent to the data repository in Lyon, France, for analysis by the collaboration.

The low level firmware code, UUB operating system code, UUB DAQ code, and code for the control micro-processor are all stored in appropriate software repositories that provide access for the collaboration. For example, the firmware source code, along with example test programs, documentation, and the compiled "bitstream" files are made available to the collaboration via a GitHub repository ${ }^{1}$, while for historical reasons the DAQ code is stored in an SVN repository.

\section{Digital functionality of the UUB}

The major portion of the AugerPrime electronics upgrade is to replace the original UB with the UUB, which is designed to fit the existing RF enclosure, and accept the existing PMT, GPS antenna, and communications cables. The new electronics employs faster ADCs (120 MHz instead of 40 $\mathrm{MHz}$ ) with larger dynamic range (12 bits each instead of 10 bits) and a significantly more powerful FPGA. Specifically, it employs a Xilinx Zynq-7020 All Programmable SoC (Artix-7 FPGA and associated Cortex A9 Dual $333 \mathrm{MHz}$ ARM co-processor) instead of the older Altera Cyclone series FPGAs used in the original electronics. Whereas the logic code of the current FPGAs is written in an Altera specific variant of VHDL called AHDL, the logic code of AugerPrime version is primarily written in IEEE standard synthesizable Verilog. Xilinx Vivado is used for the overall framework, and for standard modules such as memories, UARTs, and processor bus interfaces. Xilinx PetaLinux runs on the embedded ARM processor.

The UUB integrates various functions (analog signal processing, triggering, calibration, GPS time tagging, slow control, and data acquisition) on a single board. The FPGA is connected to a 4 Gbit LP-DDR2 memory and 2 Gbit flash memory. The FPGA implements in programmable logic

\footnotetext{
${ }^{1}$ https://github.com/auger-prime-sde/uub-firmware
} 
basic digital functions like the readout of the ADCs, the generation of triggers, and the interfaces to the LED flasher, GPS receiver, and memories. High-level functions like the data handling and the communications with the communications radio transmitter are implemented under LINUX. The current local station software originally written in OS-9 has been ported to LINUX which expands the possible pool of experts for DAQ code development and maintenance. The speed of the upgraded CPU is more than 10 times faster than that of the current one, with a similar increase in memory. This allows more sophisticated processing in the local station. The addition of accessible trigger IN/OUT signals and high-speed USB facilitates tests both in the lab and in the field.

The current local triggers $[11,12,13]$ (threshold trigger, time-over-threshold trigger (ToT), multiplicity of positive steps (MoPS) trigger, etc) are implemented using digitally filtered waveforms to reproduce the current trigger characteristics. This allows detectors with the new electronics to behave identically to the existing detectors at the trigger level and allows deployment of new electronics during the maintenance of the current system without disturbance to the data taking. The increased local processing capabilities allow new triggers to be implemented such as asymmetry-based triggers, and combined SSD and WCD triggers. The current muon memories and scalers are retained. The form factor of the UUB printed circuit board is the same as that of the UB, which allows UUB to be installed in the current enclosure, only requiring a new front panel.

Synchronization of the detectors is provided by tracking variations of the local $120 \mathrm{MHz}$ clock with respect to the GPS 1 PPS signal. For the upgraded electronics we have selected the Synergy SSR-6TF timing GPS receivers. This receiver is functionally compatible with the Motorola Oncore UT+ GPS one that is used with the current electronics. The fundamental architecture of the timetagging firmware module parallels the time-tagging design concept used in the current electronics and is implemented in the UUB board FPGA. The on-board software for initialization of the timetagging modules, GPS hardware control, and timing data is similar to the current one, with minor modifications needed for the new UUB hardware. The intrinsic GPS device accuracy after the applied granularity correction (the so-called negative sawtooth) is $\approx 2$ nanoseconds, and thus makes a negligible contribution to the overall timing accuracy.

Two digital connectors are provided for additional detectors. These connectors provide 8 differential LVDS lines, each of which can be individually defined as input or output in the FPGA. One of these is use for the radio detector, and the other is used for AMIGA. This connector also provides unregulated $+24 \mathrm{~V}$, switched and limited, with a current monitor.

\section{Analog functionality of the UUB}

A design goal of AugerPrime is to measure shower properties at energies above $6 \times 10^{19}$ $\mathrm{eV}$ as close as $250 \mathrm{~m}$ from the shower core. For this purpose the WCD is equipped with an additional small photomultiplier tube (SPMT), a 1 inch Hamamatsu R8619 PMT, dedicated to the measurement of large signals. The SPMT signal is also digitized with 12 bits at $120 \mathrm{MHz}$ in a separate channel. The SPMT gain and the amplification are set such that the dynamic range is extended by a factor of 30 to 20,000 VEM (vertical equivalent muon). This is shown in Fig. 2. The dynamic range for the SSD is 20,000 MIP (minimum-ionizing particle).

Even before PMT saturation, the dynamic range of the signals from the large XP1805 PMTs (LPMTs) and the SSD PMT is larger than can be measured by a single ADC on the UB or UUB. In 
the current electronics (UB) the anode channel of each PMT is fed to one input (low gain), while the signal from the last dynode is amplified on the PMT base to before being provided to a 2 nd input (high gain) for each PMT. The ratio between the low and high gain signals is $\approx 32$, and is calibrated using background muons in the field.

The amplified dynode signal currently used, however, is too noisy for the increased dynamic range of the UUB. Therefore the high and low gain signals are both derived from the anode signal using 2 parallel amplifiers with different gains. This has a further advantage that the low/high gain ratio is determined solely by the electronics and no longer needs to be determined from online calibrations. The signals of the 3 large PMTs are split with a gain ratio of 32 into 2 ADC channels each. The SSD PMT signal is also split, but with a gain ratio of 128 into 2 ADC channels. Fig. 3 displays a check of the ratio of the high gain to low gain channels in one station. The gain ratio is within $0.3 \%$ of the nominal ratio of 32 , as expected with the use of $0.1 \%$ gain setting resistors.

The pulse response of the XP1805, when expressed in terms of bandwidth, is $\approx 70 \mathrm{MHz}$. This is well matched to a $120 \mathrm{MHz}$ ADC and associated $60 \mathrm{MHz}$ Nyquist filter. We have chosen to use commercial 12 bit $120 \mathrm{MHz}$ AD9628 ADCs, which achieve this performance with minimal power consumption, an important consideration due to the nominal $10 \mathrm{~W}$ station power budget. The 7-pole differential Bessel filter is implemented using passive components to minimize noise contribution.

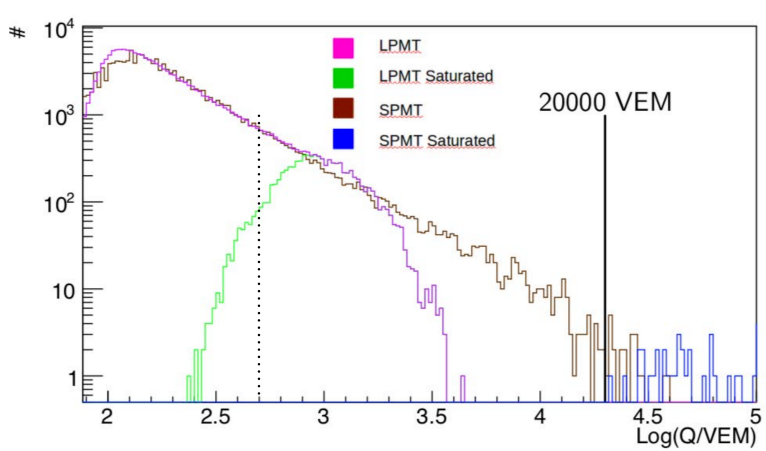

Figure 2: Extension of the dynamic range to 20,000 VEM using the small PMT. Here LPMT refers to the XP1805 PMTs, SPMT to the R8619 PMT, and "saturated" to signals which saturate the respective ADCs.

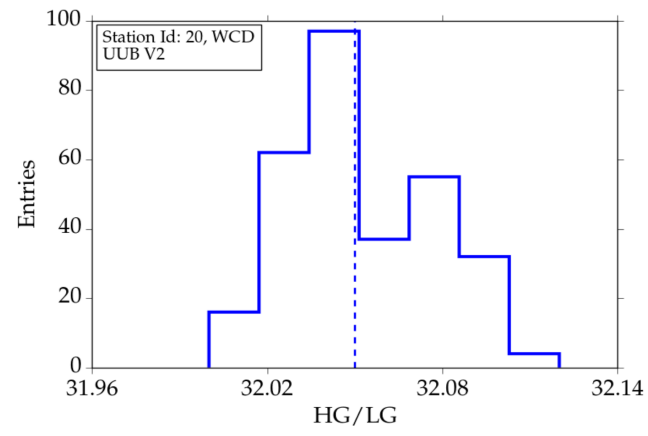

Figure 3: Example of check of the ratio of high gain to low gain channels.

\section{UUB slow control functionality}

The UUB is equipped with a micro-controller (MSP430) for the control and monitoring of the PMT's high voltage, the supervision of the various supply voltages and reset functionality. The power-on sequence of the several supplies for the FPGA is quite complex, and this is controlled by the micro-controller. It controls 16 logic I/O lines, steers a DAC (digital-to-analog converter) with eight analog outputs and senses through multiplexers up to 64 analog signals with its internal ADC. The MSP430 also provides a USB interface which can be used to monitor and control the various power supplies through a command line interface. More than 90 monitoring variables - including currents and voltages of the power supply and the PMTs are managed by the slow-control software. 


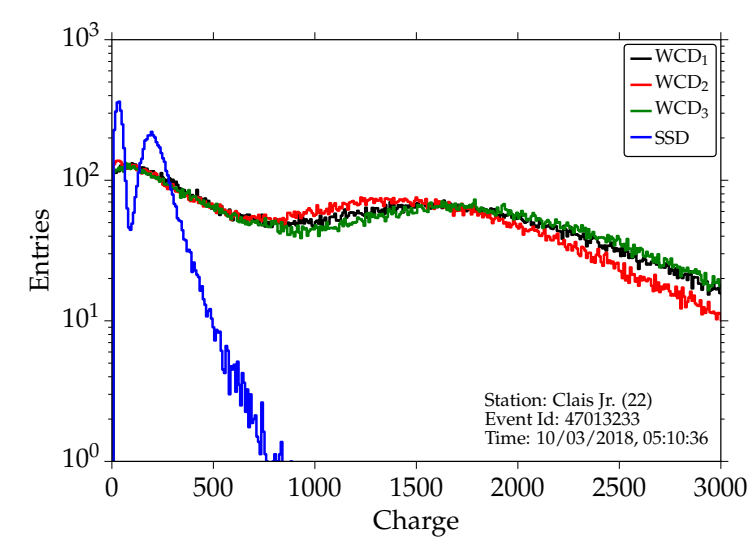

Figure 4: Example of calibration histogram of an EA detector. Calibration histograms for the previous minute are provided for each event that is read out.

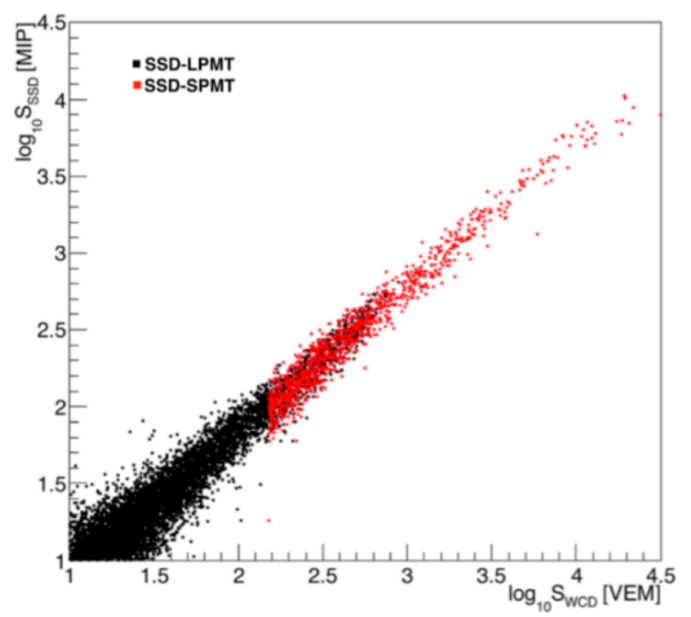

Figure 5: Correlation of signals in the SSD vs the WCD. The LPMTs are used up to saturation (black dots). The measurements are extended by means of the SPMT (red dots).

This information is then packaged by the DAQ system running on the FPGA's ARM processor and sent through CDAS to the Auger monitoring database.

\section{Calibration}

The VEM signal is the reference unit of the WCD high-gain calibrations, and was previously determined on a test tank with an external trigger hodoscope to give on average 95 photoelectrons at the cathode of the XP1805 PMTs. This corresponds to $\approx 1500$ integrated ADC counts above pedestal after signal digitization on the UUB. Due to its small area, the SPMT cannot be calibrated using astmospheric muons. A selection of small showers allows us to cross-calibrate it with the VEM signals of the LPMTs. The SSD calibration is based on the signal of a minimum-ionizing particle going through the detector. About $40 \%$ of the calibration triggers of the WCD produce a MIP in the SSD. An example of the VEM and MIP calibration histograms is shown in Fig. 4. The very good correlation between the calibrated signals of the WCD and SSD is shown in Fig. 5.

In addition to routine calibrations with physics events, each WCD is equipped with two LEDs. While these are not stable sources of calibrated light, they are useful for monitoring and linearity tests. These LEDs are also used for the SSD prototypes, but are not planned for all SSD stations.

\section{Current status and performance}

Two prototype iterations (+ retrofits) have been produced and extensively exercised in the AugerPrime EA [14]. Fig. 6 displays the RMS noise performance of a V2 UUB measured in the EA. The measured noise has already reached the level estimated using PSpice calculations.

The timing resolution has been measured using showers triggering 2 nearby stations in the AugerPrime EA. This is shown in Fig. 7. The end-to-end single station timing accuracy in real showers, including the effects of shower front fluctuations and quantization due to the $120 \mathrm{MHz}$ 


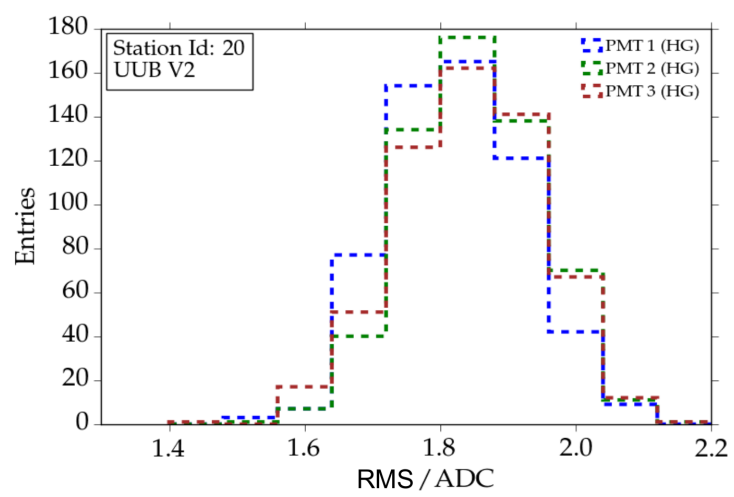

Figure 6: Measured RMS noise of a V2 UUB in the field. The performance is consistent with PSpice simulations of the noise.

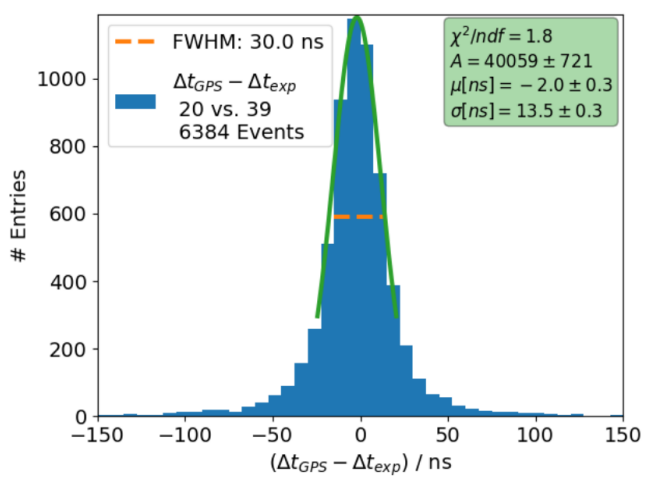

Figure 7: Measured timing resolution on showers using 2 nearby stations in the AugerPrime EA. Single station resolution is $13.5 / \sqrt{2}=9.5 \mathrm{~ns}$.

system clock, has been measured by comparing nearby stations to be better than $10 \mathrm{~ns}$, a significant improvement over the current $13 \mathrm{~ns}$.

For additional information please see Ref. [14].

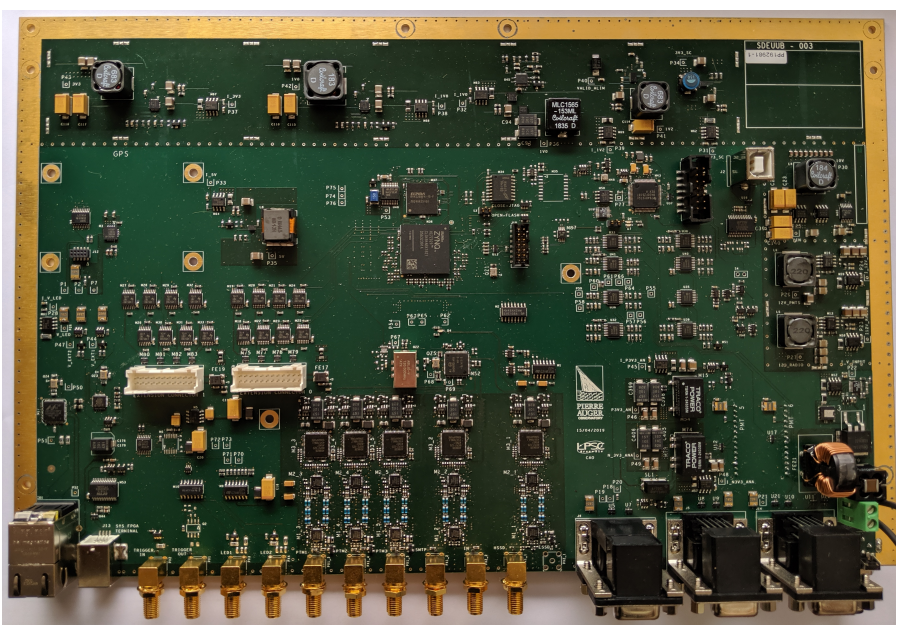

Figure 8: Photo of a V3 UUB. Shielding cans which will be installed over the DC-DC converters are not shown in this photo.

The power consumption of the UUB was targeted to be $10 \mathrm{~W}$. The actual power consumption is $12 \mathrm{~W}$. However, this turns out to not be an issue as the addition of the RD, which was not included in the original AugerPrime design, necessitates in any case an associated upgrade of the solar power system, which has sufficient capacity for both the UUB and the RD.

Based upon the experience with the V1 and V2 prototypes a V3 UUB design, incorporating small improvements, and especially improved shielding of emitted RFI, has been implemented. A photo of a V3 UUB is shown in Fig. 8. Production of the full batch (enough pre-production + production units to instrument the full array + spares) of UUBs has been initiated.

\section{References}

[1] Pierre Auger Collaboration, P. A. Collaboration, "The Pierre Auger Cosmic Ray Observatory," Nucl. Instrum. Meth. A798 (2015) 172-213. 
[2] Pierre Auger Collaboration, D. Martello, “The Pierre Auger Observatory Upgrade," in The Pierre Auger Observatory: Contributions to the 35th International Cosmic Ray Conference (ICRC 2017), pp. 147-154. 2017.

https://inspirehep.net/record/1618430/files/1617990_147-154.pdf.

[3] Pierre Auger Collaboration, A. Castellina, "AugerPrime: the Pierre Auger Observatory Upgrade," EPJ Web Conf. 210 (2019) 06002, arXiv:1905.04472 [astro-ph.HE] .

[4] Pierre Auger Collaboration, A. Aab et al., "The Pierre Auger Observatory Upgrade - Preliminary Design Report," arXiv:1604.03637 [astro-ph.IM] .

[5] Pierre Auger Collaboration, B. Pont, "A large radio detector at the Pierre Auger Observatory measuring the properties of cosmic rays up to the highest energies," in PoS(ICRC2019)395, these proceedings. 2019.

[6] Pierre Auger Collaboration, A. Aab et al., "Observation of inclined EeV air showers with the radio detector of the Pierre Auger Observatory,” JCAP 1810 no. 10, (2018) 026, arXiv:1806.05386 [astro-ph. IM].

[7] Pierre Auger Collaboration, A. Castellina, "The dynamic range of the AugerPrime Surface Detector: technical solution and physics reach," in The Pierre Auger Observatory: Contributions to the 35th International Cosmic Ray Conference (ICRC 2017), pp. 161-168. 2017. https://inspirehep.net/record/1618432/files/1617990_161-168.pdf.

[8] Pierre Auger Collaboration Collaboration, A. Aab et al., “The Pierre Auger Observatory: Contributions to the 33rd International Cosmic Ray Conference - The AMIGA muon detectors of the Pierre Auger Observatory: overview and status (ICRC 2013)," arXiv:1307.5059 [astro-ph.HE].

[9] Pierre Auger Collaboration, T. Suomijarvi, "New electronics for the surface detectors of the Pierre Auger Observatory," in The Pierre Auger Observatory: Contributions to the 35th International Cosmic Ray Conference (ICRC 2017), pp. 155-160. 2017. https://inspirehep.net/record/1618431/files/1617990_155-160.pdf.

[10] Pierre Auger Collaboration, Z. Zong, "First results from the AugerPrime engineering array," in The Pierre Auger Observatory: Contributions to the 35th International Cosmic Ray Conference (ICRC 2017), pp. 177-184. 2017. https://inspirehep.net/record/1618434/files/1617990_177-184.pdf.

[11] D. Nitz, "Triggering and data acquisition systems for the Auger observatory," IEEE Trans.Nucl.Sci. 45 (1998) 1824-1829.

[12] Pierre Auger Collaboration, D. Nitz, "The front-end electronics for the Pierre Auger Observatory surface array," IEEE Trans.Nucl.Sci. 51 (2004) 413-419.

[13] Pierre Auger Collaboration, J. Abraham et al., "Trigger and aperture of the surface detector array of the Pierre Auger Observatory," Nucl.Instrum.Meth. A613 (2010) 29-39, arXiv: 1111.6764 [astro-ph. IM] .

[14] Pierre Auger Collaboration, A. Taboada, “Analysis of Data from Surface Detector Stations of the AugerPrime Upgrade,” in PoS(ICRC2019)434, these proceedings. 2019. 